\title{
Optimization and Control of the Urban Spatial Dynamics
}

\section{Ferdinando Semboloni}

Department of Town and Regional Planning and Center for the Study of Complex Dynamics, University of Florence, Florence, Italy

\section{Key Words}

Optimization · Complex systems, control · Urban dynamics $\cdot$ Urban planning

\begin{abstract}
Urban planning involves the assignment of land use to each land cell. This process coexists and may conflict with the complex self-organized dynamics of the urban system, which should be constrained by the plan. The purpose of this study is the identification of a method for attaining the planned goals through the utilization of the self-organized dynamics, and the minimization of the constraints. The paper is organized in three steps. First, the urban plan is defined as a process of optimization. Second, the set of optimal solutions is compared with the configurations resulting by the self-organized dynamics. Third, a method for the convergence of the self-organized dynamics with the optimal configuration is proposed. In conclusion, the study shows that planning a complex system may be a hard task, while the control and the utilization of the self-organized dynamics help in the attainment of a total utility.
\end{abstract}

Copyright () 2005 S. Karger AG, Basel

\section{Fax +4161306 1234 E-Mail karger@karger.ch www.karger.com}

KARGER (c) 2005 S. Karger AG, Basel 1424-8492/05/0024-0204 $\$ 22.00 / 0$

Accessible online at: www.karger.com/cpu
Ferdinando Semboloni

Dipartimento di Urbanistica e Pianificazione del Territorio Via Micheli, 2, IT-50121 Firenze (Italy)

Tel. +39055 503 1107, Fax +39055 587087

E-Mail semboloni@urba.arch.unifi.it

\section{Simplexus}

The notion that cities have a life of their own' - that their evolution and growth seem to have something autonomous and organic about it - can be traced back at least as far as Lewis Mumford's influential book The Culture of Cities in the 1930s. And it was proposed as early as the 1960s by the urban theorist Jane Jacobs - notably in her prescient book The Death and Life of Great American Cities (1961) - that these processes might involve what complexity theorists now characterize as self-organization. But it is only relatively recently that urban geographers and planners have started to absorb the implications of these ideas. In particular, that traditional view that cities can and should be 'planned' at all seems to sit uncomfortably with the possibility that they may grow and adapt according to their own 'natural' laws. If the latter is the case - and recent work on the spatial structure and dynamics of urban centres suggests that it might be - then how can urban planners hope to superimpose any kind of policy, regulation or governance on the ways cities develop?

This is the dilemma that Ferdinando Semboloni confronts. He begins by recognizing that self-organization of urban growth may operate in opposition to the aim of urban planners to impose a topdown structure. He asks whether it is possible to find solutions that are in some sense optimal compromises between what 'comes naturally' for cities and what planners might wish to achieve, so as to guarantee efficiency and economic health in an urban environment.

Why do cities self-organize? There is still no consensus on how this process happens, or what key factors are involved, or what kind of urban structure it produces. Yet it is not hard to see why it should happen. For example, business development tends to occur on empty land, which stimulates the construction of new transport and communication infrastructure, attracts service industries, and eventually 


\section{Introduction}

Urban strategic planning has to deal with two main streams of problems arising from the widely recognized self-organizing character of the urban dynamics [1]. On one hand, the chaotic behaviour highly dependent on initial conditions of the selforganizing system has quite unpredictable effects on the planning policy [2]. On the other hand, urban planning is questioned by the ability of the urban system to steer itself [3]. While classic urban planning seeks to regulate the urban structure with a top-down approach, this evolves with the interplay of a lot of local actions [4]. In this way these two processes, even if coexistent in a city, may conflict. One solution to this problem of topical interest in urban planning is a just-in-time method, as opposed to a just-in-case one, where projects are delimited in both space and time and coupled with a constant re-evaluation of the whole sketch [5].

In this paper we choose a different way. We suggest the utilization of this self-organizing character in order to achieve the planning objectives more easily. The proposed method includes: (1) the definition of the plan as an optimization process, (2) a comparison of the optimal solutions with the configurations emerging from the self-organized dynamics, and (3) the convergence of the self-organized dynamics with the optimal configuration. In a first step this method is applied to a system with two land uses, and in the second step a more realistic situation is utilized in order to show the possible utilization of the proposed method.

\section{Optimization}

Even if urban planning is a complex process involving many actors which bargain for urban projects, it ends with the assignment of land uses or activities to land parcels, so that the public utility, however defined, is also attained. The problem of the optimal spatial assignment of activities was formulated by Koopmans and
Beckman [6]. Later the optimization process was proposed in the context of rational urban planning $[7,8]$, as well as in architectonic design [9]. Recently new methods for the research of the global optimum such as genetic algorithm have been utilized in order to establish the set of efficient alternatives in a multi-objective optimization $[10,11]$. This multi-objective optimization method refers to a theory of urban planning in which a set of efficient alternatives, or Pareto optima, are proposed to the decision makers. In other words the optimization method is utilized as an aid to the decision process, and the efficient solutions are taken as alternatives to be bargained among the decision makers [12].

In order to analyze the relation between the urban plan and the self-organized dynamics, we first consider the plan as an optimization process. The land use planning is an assignment of land use to land parcels so that a total utility function, in which the spatial relations among land uses are included, is maximized. To formalize the problem, let us suppose a set of possible land uses or states of a cell $i$ of a squared grid having $n$ cells on each side. Hence $s_{i}=$ $\{h, r, \ldots\}$, the state of cell $i$, represents the land use assigned to a cell $i$ (for instance housing, retail, etc.) and $N_{h}$ is the total number of cells in state $h$ which is established as a constraint to the optimization. In addition, for each cell $i$ a neighbourhood $\Phi_{i}$ is established, and a utility $u_{h i}$ of land use $h$ in cell $i$ which depends on $h$, and on the land uses localized in the $\Phi_{i}$ neighbourhood, as in the following equation:

$$
u_{h i}=f\left(h, s \Phi_{i}\right),
$$

where $u_{h i}$ is the utility of land use $h$ in cell $i$, and $f$ is a function. This function may take many forms. In this paper the simplest has been chosen, i.e. a linear combination, which simulates a perfect substitution of factors. Other functions with imperfect substitution of factors giving different results are not analyzed in this paper.According to the linear combination method, the encourages the development of residential areas. All of these things are interdependent. A road linking two districts may attract development in the area in between. Business and housing attract more business and housing: there are correlations between such developments. This means that, even if city growth looks amorphous, it is not random: indeed, models of correlated aggregation, imported from condensed-matter physics, have successfully described some of the characteristics of urban geography.

Semboloni begins by building an idealized model of urban land use. He considers a land surface divided into identical cells, each of which can be assigned one of a variety of uses - for example, residential, industrial or green space. The key point is that the utility of a given use for any given cell - the extent to which that particular function makes effective use of the land depends not only on its intrinsic nature but on how the surrounding land is used. To take two extreme, contrasting cases: a factory in the middle of parkland not only is detrimental to the environment but is also too isolated to be efficient, while a tiny park in the middle of an industrial estate is unlikely to draw many visitors, and thus to serve its intended function.

So Semboloni proposes that the utility $u$ of a cell $i$ assigned a land use $h\left(u_{h i}\right)$ is a function both of $h$ and of the nature of the neighbouring cells. This function could take many forms; he assumes one of the simplest, which is that the utility for cell $i$, with use $h$, for contribution to $u_{h i}$ from a neighbouring cell $j$ to be devoted to use $r$, is simply a constant $m_{h r}$ that depends on $h$ and $r$.

The question is then: what maximizes the total utility, the sum of $u_{h i}$ over all cells? In a planning process, this depends on what one assumes about the relative importance of different types of land use $h$ planners typically want to ensure that a given proportion of land is assigned to residential use, for example, and to ensure 
utility is calculated as in the following expression:

$$
u_{h i}=\sum_{r}\left(m_{h r} \sum_{j \in \Phi_{i}} r_{j}\right),
$$

where $r_{j}=1$ if the use $r$ is assigned to cell $j$, otherwise $r_{j}=0$. Further $m_{h r}$, an element of the matrix $\mathbf{M}$, is the utility for use $h$ to be surrounded by use $r\left(\Sigma_{r} m_{h r}=1\right)$. In words, equation 2 states that utility depends on the quantity of the land uses in the neighbourhood multiplied by a parameter. The total utility of each land use is:

$$
U_{h}=\sum_{i} u_{h i} .
$$

Moreover, a weight is assigned to each land use in order to calculate the total utility $U_{T}$, as in the following equation:

$$
U_{T}=\sum_{h} W_{h} U_{h} .
$$

This $W_{h}$ weight $\left(\sum_{h} W_{h}=1\right)$ represents the economic capacity of the land use $h$ to bid for the land, or the importance assigned to this land use in case a plan is implemented. This is an usual assumption in multicriteria decision making, and it is the simplest method to compare the otherwise incommensurable vectors of the utilities. The planning problem is stated as the research of the assignment which maximizes the total utility, for each possible set of weights. By using this method, under the assumption of the previous equation 4 , one obtains the set of Pareto optimal solutions [13]. For each of these solutions it is impossible to improve the utility of a land use $U_{h}$ without decreasing the utility of another land use $U_{r}$.

In the following experiments the maximization of utility is obtained by using the simulated annealing method [14]. Not only is this a general optimization method which can be applied to every sort of problems, but it is formulated as a simulation of a dynamic process which can be easily compared with the self-organized dynamics. When the simulated annealing method is applied, the process begins by using a random pattern of land uses. Then, at each step one couple of cells is taken at random, the land uses are exchanged, and the total utility $U_{T}$ of the new pattern is evaluated by using equation 4 . A new configuration is considered or not, according to the method of simulated annealing which decreases the temperature, thus allowing the system to reach and maintain a stable state in which the energy $E=-U_{T}$ is minimized, and the total utility is maximized.

Nevertheless, the urban system is usually able to reach some stable state by using its self-organizing character. In this case the maximization of the total utility is not a necessary, even if a possible [15] outcome of the process. We call this process self-organized dynamics and present it in the following section.

\section{Self-Organized Dynamics}

A lot of studies on the micro-simulation of the urban dynamics are available. Usually a cellular automaton framework is utilized, even if an agent-based modelling approach is emerging as a novel method [16]. For the most part these approaches consider an expanding system from a central seed. Since we are more interested in the internal rearrangement of the system than in its growth, a different approach is utilized, which is partially similar to that utilized in the simulated annealing method. In fact beginning from a random pattern of the established land uses, at each step two cells are chosen at random and the land uses are exchanged if this exchange does not decrease the sum of the utilities of the two land uses. Thereby the land use $h$ in cell $i$ is exchanged with the land use $r$ in cell $j$ if:

$$
u_{r i}+u_{h j}>u_{h i}+u_{r j} \text {. }
$$

This method simulates spatial dynamics in which the local individual utility is maximized, as in a market in which a transaction happens only if a couple of individuals think that they will be more sat- some green space even though this is economically less productive than industrial space. So the utilities are summed according to some weighting $W$ for each $h$.

In principle, given the set of $m_{h r}$ values, the maximum utility can be calculated by some optimization method, such as simulated annealing: the problem is somewhat akin to that of a spin glass, in which one seeks the most stable orientation of atomic spins subject to the constraints of their interactions with neighbours.

But what if one were to put aside concerns about global optimization and to consider how the system of cells evolves purely on the basis of local interactions between them? This is what gives rise to selforganization, and is best modelled using a cellular automata procedure. Semboloni looks at the dynamics that will emerge on the basis of a simple rule: two cells are chosen at random, and their uses exchanged. The change is accepted if the sum of local utilities of the two cells is not lowered thereby. No account is now taken of the global utility, which may increase or decrease as a result. This looks more like how real urban space evolves: it is determined by local gains rather than by reference to the overall urban landscape.

The ideal outcome would be for the selforganizing process to produce the same result as the globally optimal one. But there is no guarantee that this will be so, and indeed the two solutions will differ in general. So how can one make them converge?

The conventional planning approach, says Semboloni, is simply to penalize land use that does not conform to the optimal solution, and thus to try to impose the optimal configuration by regulation. His alternative is less directive - he suggests that the self-organized solution might be guided towards the optimal one by 'pinning' just a few key cells to certain land uses, so that they might act as catalysts that help the dynamic urban pattern converge on the optimum. The aim is then to identify 
isfied after than before a transaction. In addition this method mimics the efforts for establishing a solution through a lot of repeated trials, like in the simulated annealing method. But, in this case at each exchange, the sum of the local utilities is maximized instead of the total utility $U_{T}$ which may both increase or decrease because the utility of the surrounding cells is not considered. The stability of this selforganized dynamics is attained when for each couple of cells the exchange does not increase the sum of the utilities of the two land uses.

The Utopian situation corresponds to the similarity between the optimal configuration and the result of the self-organized dynamics. In this case the utilities obtained with the optimization process and with the self-organized dynamics are equivalent in the steady state. This comparison allows to divide the set of Pareto solutions into two subsets: the A set of solutions in which the optimum is similar to the outcome of the self-organized dynamics and the B set for which there is no similarity. This similarity is evaluated comparing the utility attained by the optimal configuration in relation with the utility attained by the self-organized dynamics. Thereby, an index of dissimilarity $I^{d}$ is defined:

$$
I^{d}=\frac{U_{T}^{1}-U_{T}^{2}}{U_{T}^{1}},
$$

where $U_{T}^{1}$ is the total utility attained with the optimal configuration and $U_{T}^{2}$ is the total utility attained with the self-organized dynamics. This index ranges from zero, because $U_{T}^{1} \geq U_{T}^{2}$, to 1 .

\section{Convergence of the \\ Self-Organized Dynamics with the Optimal Configuration}

In case the optimal solution and the self-organized dynamics do not match, one would like to make the self-organized dynamics convergent with the optimal configuration. The classic urban planning approaches this problem by establishing a feedback for each point of the urban surface. In case the land use in an urban zone does not agree with the established use, and this mismatch is officially observed by the control agency, then a sort of penalty, usually established by the law, is applied in order to re-establish the planned assignment. This is the principle of the dynamic system control, in which there is a master system, the plan, and a slave system, the urban dynamics, and each point of the master system is coupled with the corresponding point of the slave system. This method simply constrains the self-organized dynamics into the optimal configuration. The energy spent in this process by the control agency depends on the distance between the urban plan and the result of the selforganized dynamics. For this reason an urban plan is often conceived in a way that this distance is reduced in order to make the desired configuration more attainable.

In contrast to the classic urban planning, the proposed method is based on the utilization of the self-organizing characteristic of the urban system and on the minimum number of cells whose established land use is not allowed to change during the simulated dynamics. These pinning cells play the role of control but also of catalysts accelerating positive effects in the urban dynamics [17] without themselves being transformed. The identification of the minimum number of pinning cells which is able to make the self-organized dynamics convergent with the optimal configuration is the core of the problem. This aspect has been widely studied in the field of the control of chaotic spatiotemporal systems $[18,19]$, and in essence consists in the identification of the cells which are strategic for the control of other cells in the spatial dynamics.

To establish the minimum number of cells able to control the urban dynamics, these are sorted according to the index of influence. The method utilized to calculate the index of influence of a cell is based on these key cells, which he does by defining a method for calculating their degree of influence on the global pattern, $I^{i}$.

To test this process, Semboloni begins with a simple case: a $10 \times 10$ grid, with just two types of land use, housing $(h)$ and retail $(r)$. He considers three different sets of rules for how adjacent land uses affect one another - that is, three different sets of values of $m_{h r}$. Each land use is constrained to occupy half of the grid. For the first two rules, the optimal and self-organized solutions are very similar, and so no 'guiding' of the dynamics is needed to make the two converge. The third rule does generate discrepancies, and here the assignation of 'pinning cells' can reduce the difference between the two solutions - although it falls to zero only when half the total number of cells are pinned, in effect enforcing the optimal outcome by fiat.

But in more complex cases, pinning seems to be easier. For example, in the case where 50 cells are assigned to housing, 10 to retail, and 40 to open space, the retail cells turn out to be central to the overall pattern - so pinning these alone is sufficient to guarantee good convergence in most cases. Much the same applies when the number of land uses, or the number of cells, is increased still further: it turns out that particular land uses tend to exert a dominant influence on the spatial partitioning, so that just a few influential cells pinned to this use can control the outcome.

This sounds like good news for planners, because it suggests that it is not necessary to expend lots of resources, or to apply strong land-use regulations over large areas of urban space, in order to steer development towards a desired goal. Rather, it may be feasible to identify the crucial 'catalytic sites' that will help a city to evolve spontaneously towards the state that maximizes its efficiency and potential.

Philip Ball

p.ball@nature.com 
a kind of input-output analysis. In fact in the optimal configuration each cell generates and receives utility from the bordering cells. According to equations 2 and 4, this utility $v_{i j}$ generated by the land use $r$ in cell $i$ in relation to the land use $h$ in cell $j$ is defined in the following way:

$$
v_{i j}=W_{h} m_{h r} .
$$

By using the previous equation, the matrix $\mathbf{G}$ of the exchanged utility is calculated. Each element $g_{i j}$ of the matrix is the ratio: utility generated by the cell $i$ in the cell $j$ total utility generated in cell $j$ by the whole set of cells, as in the following expression:

$$
g_{i j}=\frac{v_{i j}}{\sum_{k} v_{k j}} .
$$

In order to consider both the direct and the indirect effects the following matrix is calculated:

$$
\mathbf{G}^{*}=\mathbf{G}+\mathbf{G}^{2}+\mathbf{G}^{3}+\ldots
$$

The index of influence $I^{i}$ for each cell is then calculated as the difference between the capacity to influence $\left(\Sigma_{j} g_{k j}^{*}\right)$ minus the degree of being influenced $\left(\Sigma_{i} g_{i k}^{*}\right)$ :

$$
I_{k}^{i}=\sum_{j} g_{k j}^{*}-\sum_{i} g_{i k}^{*} .
$$

In the first step $t$ of the ordering process, the first pinning cell $k$ is chosen which corresponds to the maximum of $I^{i}$. Then we reason in the following way. If the cell $k$ is established as a pinning site, the control of the other cells immediately dependent on the $k$ cell is useless. We would prefer the second pinning cell to have a high index of influence, while being less dependent on the first. For this reason we subtract from the capacity of control of each cell $l$ a share, $g_{k l}^{*} / \sum_{i} g_{i l}^{*}$, corresponding to the share of control exercised by the $k$ cell on cell $l$. Thereby in the next step $t+1$ the elements of the matrix $G^{*}$ are recalculated in the following way:

$$
g_{l j}^{*}(t+1)=g_{l j}^{*}(t)\left[1-\frac{g_{k l}^{*}(t)}{\sum_{i} g_{i l}^{*}(t)}\right] .
$$

Further the row $k$ is set equal to zero in order to exclude the cell $k$ from being newly selected as a pinning cell, and the process is repeated until for each cell, the index related to its capacity to influence the land use of the other cells is calculated (fig. 1).

Clearly a relation should exist between the number of pinning cells and the similarity between the optimal configuration and that obtained with the self-organized dynamics. In order to analyze this relation, we define $D_{p q}$ as the distance between the configurations $p$, the plan, and $q$, the outcome of the self-organized dynamics. This distance is calculated by summing up the cells having a different land use in $p$ and $q$. By using this distance we consider the following relation:

$$
D_{p q}=f\left(C_{q}\right)
$$

where $C_{q}$ is the number of pinning cells, successively taken from the ranked list established by using the previously explained method. Usually $D_{p q}$ decreases with the increase of $C_{q}$. In fact in case all the cells are declared as pinning cells, this is like in the classical urban planning, and $D_{p q}=0$. In turn we can decide the quantity of pinning cells, beginning from the first in the ranked list and evaluate the rate at which the distance decreases, looking for the minimum number of pinning cells resulting in an acceptable distance. In order to study this and the other aspects in the next section some theoretical experiments are shown which highlight, with an increasing complexity, the relation between optimization and control in the spatial dynamics.

\section{Experiments and Discussion}

In this section the proposed method is applied to a set of theoretical cases of increasing complexity. In each case, through the variation of the weights the set of optimal solutions is obtained. Further, the relationship between this set and the corresponding results of the self-organized dynamics is discussed in order to highlight the conditions under which the control of

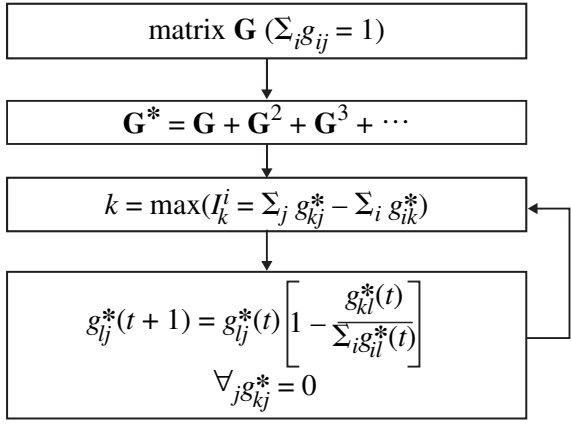

Fig. 1. Algorithm for ranking the pinning cells.

the complex system is easiest and most efficient.

In order to study the simplest situation a $10 \times 10$ lattice surface and two possible land uses were chosen for each cell: the housing $(h)$ and retail $(r)$ land use. In the first three experiments, the total quantities of each land use are constrained to 50. The rules of interaction refer to three paradigmatic cases. Integration: Each land use is attracted by other land use. Segregation: Each land use is attracted by a similar land use. Integration and segregation: The housing land use is attracted by the retail land use, while the latter is attracted only by itself. The neighbourhood $\Phi_{i}$ is limited to the eight bordering cells. The corresponding values of the elements of the $\mathbf{M}$ matrix are shown in table 1.

The segregation dynamics has been widely investigated, beginning with the work of Schelling [20], with the purpose of connecting the emergent properties of the resulting pattern with a parameter related to the degree of segregation. The self-organized dynamics here presented as a first step in the theoretical experiments is very similar to these previous models. In fact in the first two sets of experiments (integration and segregation) the two extreme cases with a low and a high degree of segregation are considered. In the third experiment (integration and segregation), through the variation of the weights as- 
Table 1. Matrix $\boldsymbol{M}$ in the three cases

\begin{tabular}{|c|c|c|c|c|c|c|}
\hline \multirow[t]{2}{*}{ Land use } & \multicolumn{2}{|c|}{ Integration } & \multicolumn{2}{|c|}{ Segregation } & \multicolumn{2}{|c|}{ Integration-segregation } \\
\hline & housing & retail & housing & retail & housing & retail \\
\hline Housing & 0 & 1 & 1 & 0 & 0 & 1 \\
\hline Retail & 1 & 0 & 0 & 1 & 0 & 1 \\
\hline
\end{tabular}

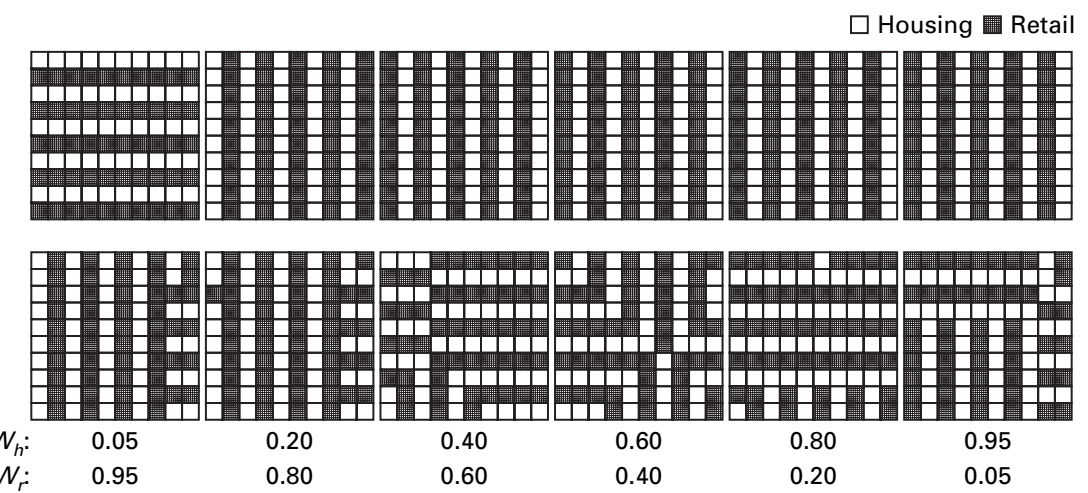

Fig. 2. Integration. Upper row shows the patterns obtained through optimization. Lower row shows the patterns obtained by applying the rules of the self-organized dynamics to a random pattern. At the bottom of each column the set of weights utilized for housing $\left(W_{h}\right)$ and retail $\left(W_{r}\right)$ is shown.

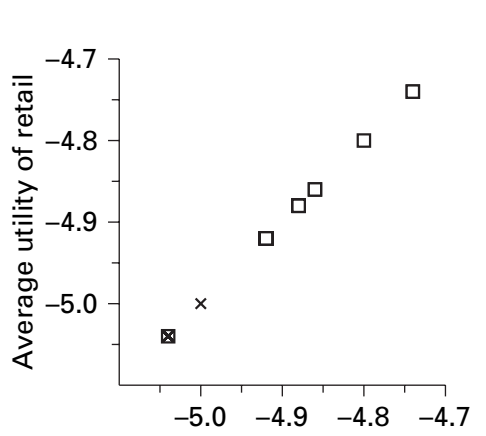

a

Average utility of housing

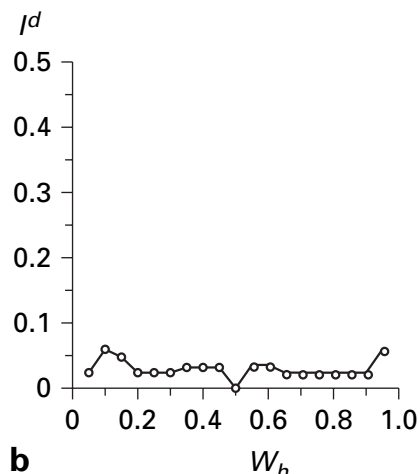

Fig. 3. Integration. a The Pareto front. $x=$ Configurations obtained through optimization; $\square=$ configurations obtained by applying the rules of the self-organized dynamics to a random pattern. $\mathbf{b}$ The variation of $l^{d}$, the index of dissimilarity, in relation to $W_{h}$, the weight applied to the housing. signed to the two land uses (one of which is totally devoted to integration and the other one to segregation) we explore the different configuration emerging, as from a variation of the degree of segregation. In turn the results of the optimization process may differ from the Schelling model, because the total utility is considered and not only that of the two exchanging land uses.

\section{Integration}

In the integration experiment, for each set of weights the outcome of the optimization is similar to that obtained with the self-organized dynamics (fig. 2), and practically all the optimal configurations belong to the A set. The resulting total utilities for each set of weights are also similar (fig. 3). This effect depends on the equal number of land uses. In fact, because the utility is generated by a couple of different cells, the number of housing cells surrounding a retail cell in the steady state is equal to the number of retail cells surrounding a housing cell. A set of rows that horizontally or vertically disposed of alternate land uses is the configuration that attains this effect, and the utility for each land use is always the same. The Pareto front is in fact represented by only one point, and the optimal configuration roughly coincides with the result of the self-organized dynamics. This is the Utopian situation in which the social utility agrees with the individual utility and in essence a plan is not necessary.

\section{Segregation}

In the segregation experiment the patterns emerging from applying the segregation rules are 2-fold: the concentration of one use in the centre and the division of space (horizontally or vertically) (fig. 4). In fact due to the finite size of the surface, it is not possible for the two land uses, at the same time, to be circular shaped. The Pareto front is thereby represented by three points: the first two corresponding to the concentration of one use in the centre and the third corresponding to the division 


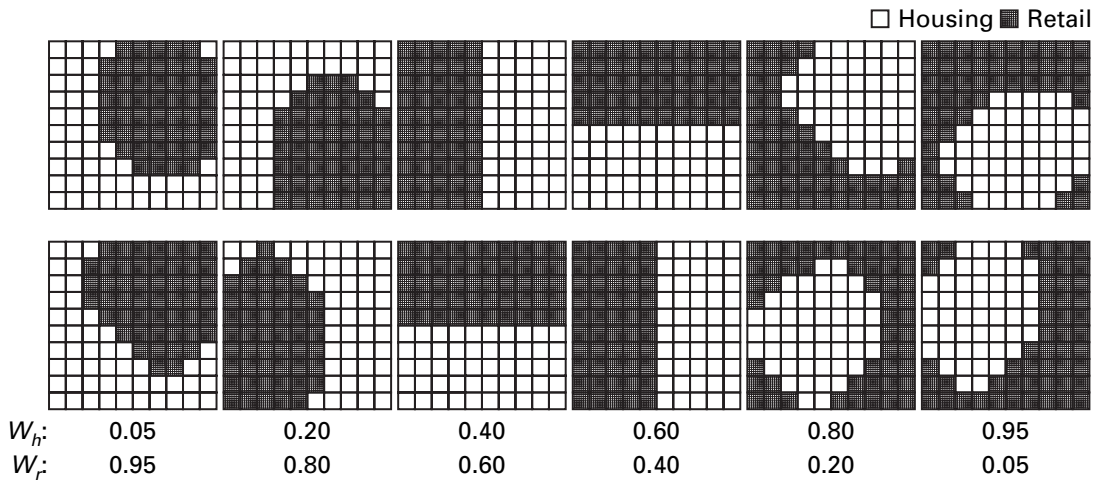

Fig. 4. Segregation. Upper row shows the patterns obtained through optimization. Lower row shows the patterns obtained by applying the rules of the self-organized dynamics to a random pattern. At the bottom of each column the set of weights utilized for housing $\left(W_{h}\right)$ and retail $\left(W_{r}\right)$ is shown

(fig. 5). When the self-organized dynamics method is applied it produces a pattern which is similar to that produced by the optimization process (fig. 4): all the optimal configurations belong to the A set. Even if the utilities of the two land uses are in opposition the situation is similar to the pre- vious one and in essence a plan is superfluous.

\section{Integration and Segregation}

The results of the integration and segregation experiment appear similar to those of the two previous experiments: both the

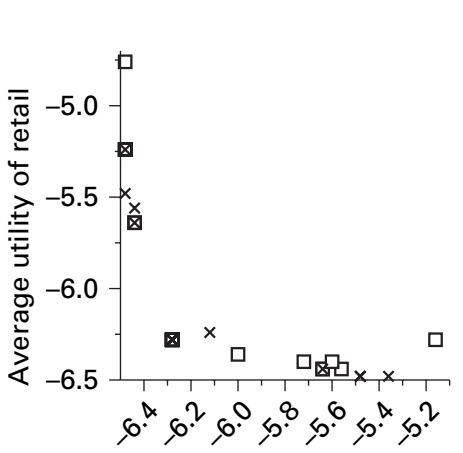

a

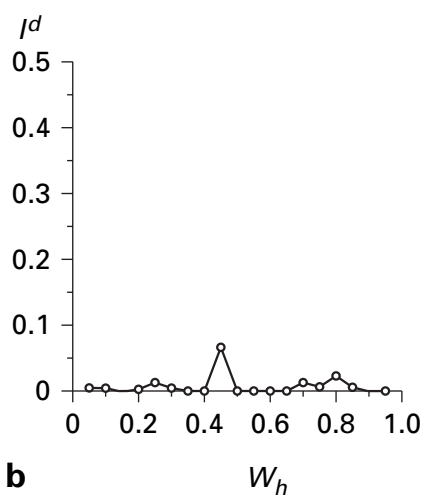

Fig. 5. Segregation. a The Pareto front. $\times=$ Configurations obtained through optimization; $\square=$ configurations obtained by applying the rules of the self-organized dynamics to a random pattern. $\mathbf{b}$ The variation of $I^{d}$, the index of dissimilarity, in relation to $W_{h}$, the weight applied to the housing. concentration of one use in the centre and the rows horizontally or vertically disposed of alternate land uses (fig. 6). In order to compare more precisely these sets of experiments, let us focus on the differences in the matrices $\mathbf{M}$. This matrix is asymmetric in this experiment while it was symmetric in the two previous ones. However, this difference is only apparent. In fact, according to equation 7, the total utility $U_{T}$ can also be calculated as the sum of the utilities related to each couple of bordering cells:

$$
u^{*} i j, h r=W_{h} m_{h r}+W_{r} m_{r h}
$$

where $u_{i j, h r}^{*}$ is the utility related to the couple of bordering cells $i$, where the land use $h$ is located, and $j$, where the land use $r$ is located, and $U_{T}=\Sigma_{i j} u_{i j}^{*}$. Further the symmetric matrix of interaction $\mathbf{M}^{S}$ is defined in which:

$$
m_{h r}^{s}=m_{r h}^{s}=\frac{W_{h} m_{h r}+W_{r} m_{r h}}{2} .
$$

The total utility can also be calculated by using this symmetric matrix, as in the following equations:

$$
u_{i j, h r}^{*}=2 m_{h r}^{s} \text {, and } u_{j, i, r h}^{*}=2 m_{r h}^{s} .
$$

Because each couple is considered twice the total utility calculated with this method equals that calculated with equation 4 . Now consider the symmetric interaction matrices $\mathbf{M}^{S}$ of the integration and of the integration and segregation experiments in relation to the set of weights: $W_{h}=0.95$ and $W_{r}=0.05$. As it is straightforward from the upper part of table 2 these two matrices are similar as well as the interaction matrices of the segregation experiment and of the integration and segregation in relation to the set of weights: $W_{h}=$ 0.05 and $W_{r}=0.95$ (lower part of table 2). This is why the pattern (fig. 6) obtained with $W_{h}=0.05$ and $W_{r}=0.95$ is similar to that obtained in the segregation experiment, while the pattern obtained with $W_{h}$ $=0.95$ and $W_{r}=0.05$ is similar to that ob- 


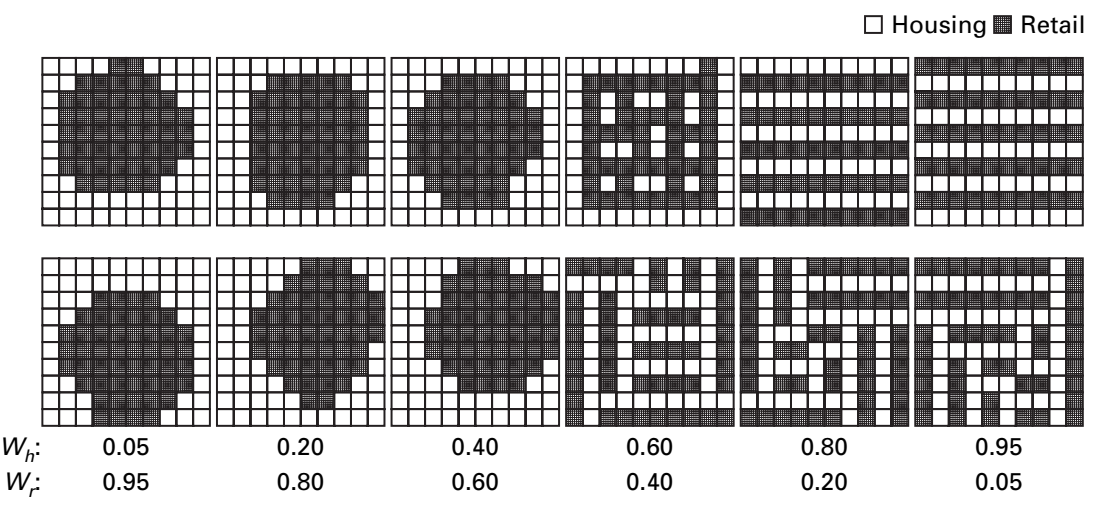

Fig. 6. Integration and segregation. Upper row shows the patterns obtained through optimization. Lower row shows the patterns obtained by applying the rules of the self-organized dynamics to a random pattern. At the bottom of each column the set of weights utilized for housing $\left(W_{h}\right)$ and retail $\left(W_{r}\right)$ is shown.

Table 2. Comparison of the matrices $M^{\mathrm{s}}$

\begin{tabular}{|c|c|c|c|c|}
\hline \multirow[t]{2}{*}{ Land use } & \multicolumn{2}{|c|}{ Integration $^{\mathrm{a}}$} & \multicolumn{2}{|c|}{ Integration-segregation $^{a}$} \\
\hline & housing & retail & housing & retail \\
\hline \multirow{4}{*}{$\begin{array}{l}\text { Housing } \\
\text { Retail }\end{array}$} & 0 & 0.5 & 0 & 0.475 \\
\hline & 0.5 & 0 & 0.475 & 0.05 \\
\hline & \multicolumn{2}{|c|}{ Segregation ${ }^{\mathrm{b}}$} & \multicolumn{2}{|c|}{ Integration-segregation ${ }^{b}$} \\
\hline & housing & retail & housing & retail \\
\hline Housing & 0.05 & 0 & 0 & 0.025 \\
\hline Retail & 0 & 0.95 & 0.025 & 0.95 \\
\hline
\end{tabular}

\footnotetext{
${ }^{\mathrm{a}} W_{h}=0.95$ and $W_{r}=0.05$.

${ }^{\mathrm{b}} W_{h}=0.05$ and $W_{r}=0.95$.
}

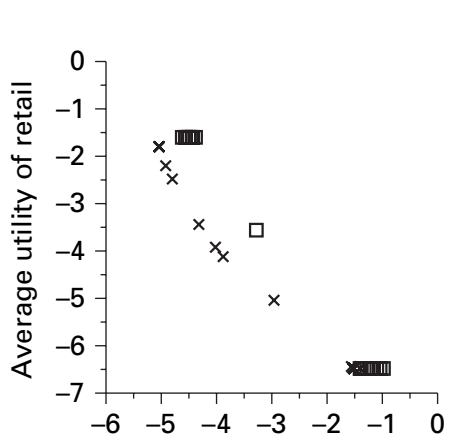

a Average utility of housing

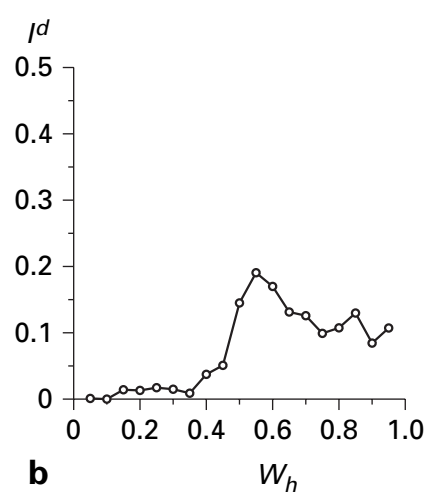

Fig. 7. Integration and segregation. a The Pareto front. $\times=$ Configurations obtained through optimization; $\square=$ configurations obtained by applying the rules of the self-organized dynamics to a random pattern. $\mathbf{b}$ The variation of $I^{d}$, the index of dissimilarity, in relation to $W_{h}$, the weight applied to the housing. tained with the integration experiment. In addition an intermediate pattern (a transition between the two) is obtained when the weights assigned to the land uses are similar.

The Pareto front is less convex than in the previous experiment, while the outcomes of the self-organized dynamics and of the optimization process are similar (A set) till the weight assigned to the retail is greater than that assigned to housing (fig. 7). Especially in the intermediate regime and when the weight assigned to the housing is greater than that assigned to retail, the optimal solutions belong to the B set.

The dissimilarity between the optimal and the self-organized configuration observed for the first time results in the applying of the method for the convergence. The outcome has been evaluated by considering the relation: number of pinning cells-distance between optimal and selforganized configuration $\left(D_{p q}\right)$. As figure 8 shows, the decrease of the distance is proportional to the number of pinning cells apart from the last two cases (fig. 8e,f). But in all the cases the distance is zero only when the number of pinning cells equals 50 , which coincides with the number of housing and retail land uses.

\section{Integration and Segregation, Second Experiment}

Because these results are influenced by the equal number of housing and retail land uses, a similar experiment was performed with a different number of land uses, and with the inclusion of the open land. The number of land uses changed as follows: 50 cells for housing, 10 for retail and 40 for open space, while the matrix $\mathbf{M}$ was slightly modified as in table 3 . Through this variation the degrees of freedom of the housing and retail land uses increase in the occupation of the surface. In fact, as figure 9 shows, the resulting patterns are quite different. The optimal configurations are 2-fold: the concentration of the retail land use in the centre, which is similar to the 

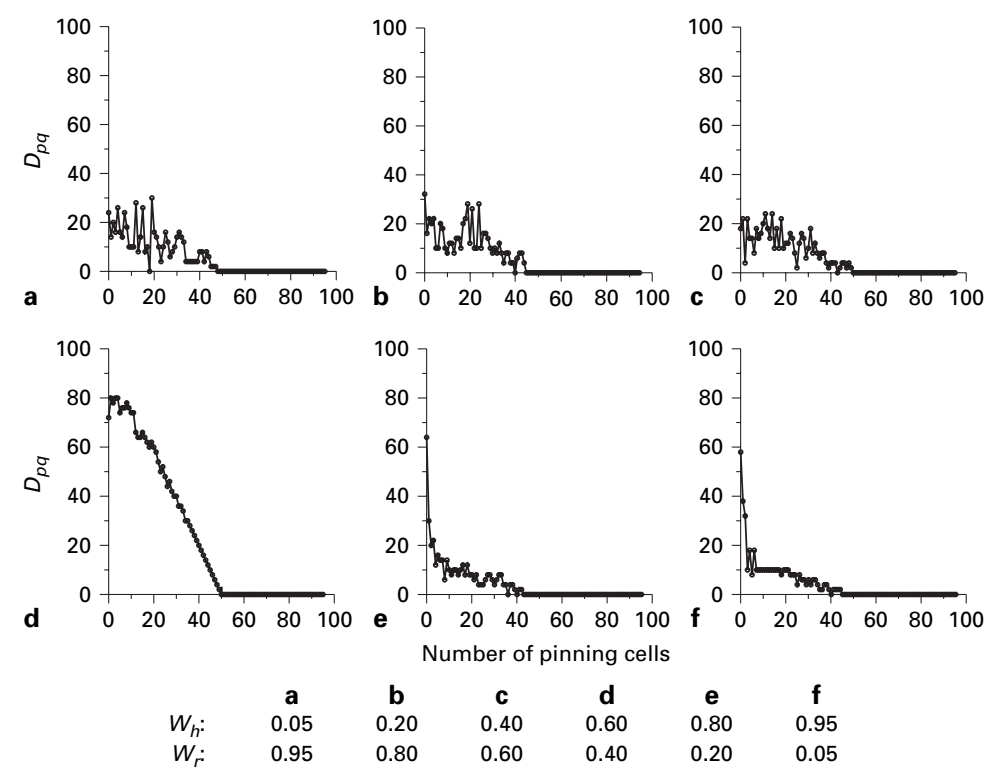

$\begin{array}{ccccc}\mathbf{b} & \mathbf{c} & \mathbf{d} & \mathbf{e} & \mathbf{f} \\ 0.20 & 0.40 & 0.60 & 0.80 & 0.95 \\ 0.80 & 0.60 & 0.40 & 0.20 & 0.05\end{array}$

Fig. 8. Integration and segregation. The varying of distance $D_{p q}$ with the increase of the number of the pinning cells in which the land use has been fixed.

$\boxplus$ Housing
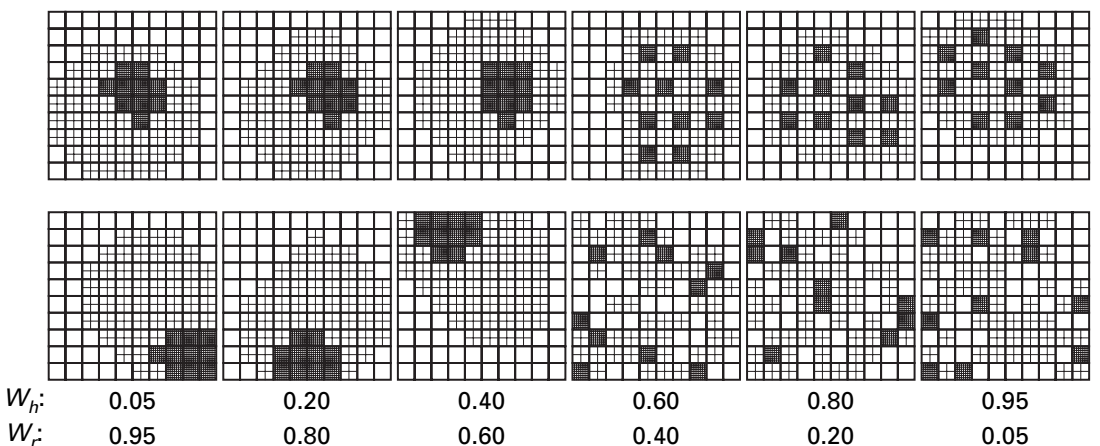

Fig. 9. Integration and segregation, second experiment. Upper row shows the patterns obtained through optimization. Lower row shows the patterns obtained by applying the rules of the self-organized dynamics to a random pattern. At the bottom of each column the set of weights utilized for housing $\left(W_{h}\right)$ and retail $\left(W_{r}\right)$ is shown.

Table 3. Integration and segregation, second experiment, matrix $M$

\begin{tabular}{lll}
\hline Land use & \multicolumn{2}{l}{ Integration and segregation } \\
\cline { 2 - 3 } & housing & retail \\
\hline Housing & 0.1 & 0.9 \\
Retail & 0 & 1 \\
\hline
\end{tabular}

result of the self-organized dynamics and a homogeneous distribution of retail regularly mixed with the housing land use which differs from the result of the self-organized dynamics. Thereby, the Pareto front is reduced to only two points (fig. 10). The index of dissimilarity $I^{d}$ increases with the increase of the weight $W_{h}$ assigned to the housing land use (fig. 10).

In turn a low number of pinning cells is enough to obtain an important decrease of the distance (fig. 11). In fact in the first ten pinning cells (see fig. 11d-f) the ten retail land uses are located, which influence the location of the housing cells.

This last aspect highlights the relation between the self-organized dynamics and the optimization process. These two processes converge when the maximum weight is assigned to a land use which plays a central role in the interaction. The centrality of a land use can be roughly calculated by using the difference: sum by columns minus sum by row in the matrix $\mathbf{M}$ (table 3 ), i.e. the capability to influence minus the degree of being influenced, as it has been done with the calculation of the influence index. It is easy to conclude that in this experiment the retail land use plays the most central role. Hence the optimal configuration and the outcome of the self-organized dynamics coincide when a big weight is assigned to the retail land use. In turn, a lot of energy has to be spent to compel the system to converge with the optimal configuration, when the maximum weight is assigned to a land use which does not play a central role, in the previously defined sense.

\section{A More Realistic Experiment}

The following experiment is conceived in a way that is less schematic and more similar to the reality. Two new land uses, industry and equipment, are added and each cell can be assigned to one of the following land uses: housing (30 cells), retail (5 cells), industry ( 10 cells), open land (53 cells), and equipment ( 2 cells). The location of equipment is fixed, in order to simulate the existence of some exogenous factors. The matrix $\mathbf{M}$ is shown in table 4 . As usual, positive effects occur for housing in case there is retail and open land uses in the nearby cells, and, to a lower degree, industry where workplaces are located. Posi- 


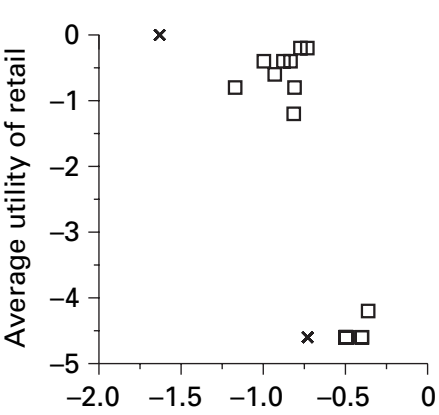

a Average utility of housing

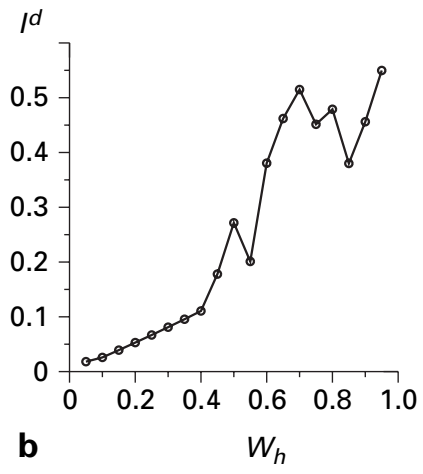

Fig. 10. Integration and segregation, second experiment. a The Pareto front. $\times=$ Configurations obtained through optimization; $\square=$ configurations obtained by applying the rules of the self-organized dynamics to a random pattern. $\mathbf{b}$ The variation of $l^{d}$, the index of dissimilarity, in relation to $W_{h}$, the weight applied to the housing.

Table 4. Matrix $M$ and number of land uses

\begin{tabular}{lllllll}
\hline Land use & Housing & Retail & Industry & Open land & Equipment & $\begin{array}{c}\text { Number of } \\
\text { land uses }\end{array}$ \\
\hline Housing & 0.3 & 0.4 & 0.1 & 0.2 & 0.0 & 30 \\
Retail & 0.35 & 0.5 & 0.15 & 0.0 & 0.0 & 5 \\
Industry & 0.0 & 0.1 & 0.2 & 0.0 & 0.7 & 10 \\
Open land & 0.0 & 0.0 & 0.0 & 0.0 & 0.0 & 53 \\
Equipment & 0.0 & 0.0 & 0.0 & 0.0 & 0.0 & 2 \\
\hline
\end{tabular}
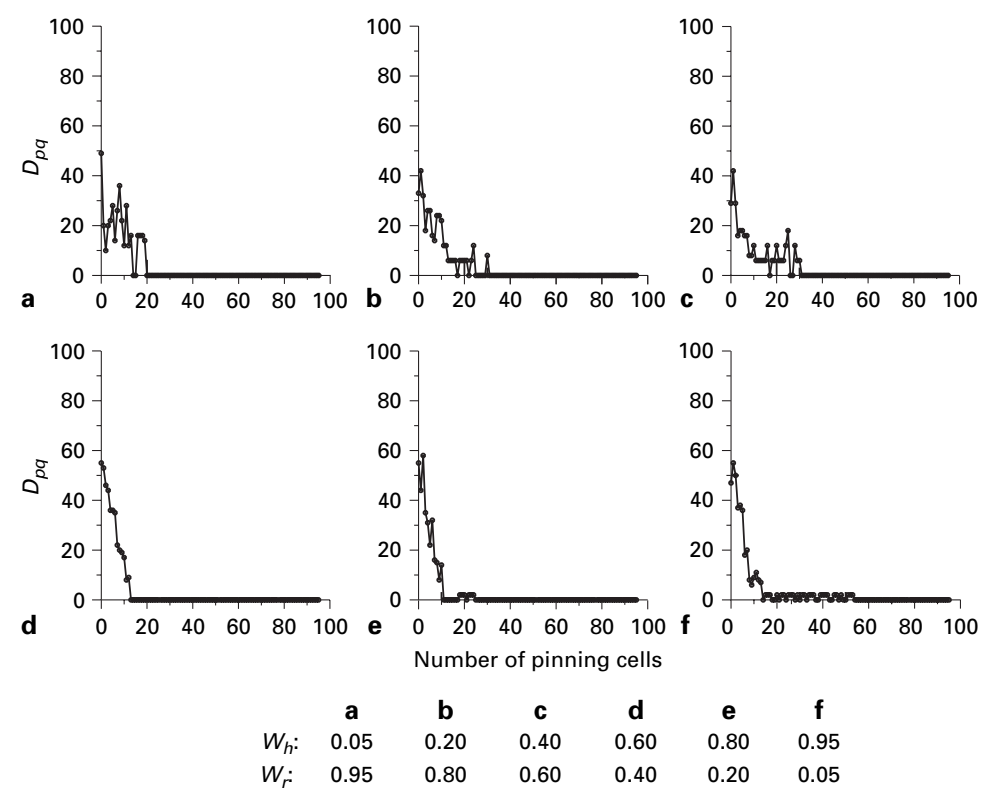

$\begin{array}{ccc}\mathbf{b} & \mathbf{c} & \mathbf{d} \\ 0.20 & 0.40 & 0.60 \\ 0.80 & 0.60 & 0.40\end{array}$

$\begin{array}{cc}\mathbf{e} & \mathbf{f} \\ 0.80 & 0.95 \\ 0.20 & 0.05\end{array}$

Fig. 11. Integration and segregation, second experiment. The varying of distance $D_{p q}$ with the increase of the number of the pinning cells in which the land use has been fixed. tive effects occur also for the retail activities in relation to housing and industry which represent in various degrees a demand for retail. Industry utility increases with the contiguity to the equipment.

Figure 12 shows the outcomes of the experiment including also the influence index of each cell, while in figure 13 the Pareto front is reported in three views. The convergence depends on the weigh assigned to the retail land use which at a first insight appears as the most central in the interaction rules. However, to calculate more rigorously the index of centrality of a land use we reason in the following way. Remembering that $m_{h r}$ is the utility for use $h$ to be surrounded by use $r, m_{r h}$ is a measure of the influence of $r$ on $h$. The more land $h$ uses, the higher is the absolute influence of $r$. For this reason a matrix $\mathbf{M}^{\prime}$ is defined which is the transpose of the matrix $\mathbf{M}$, where each element is multiplied by the probability to find the influenced land use:

$$
m_{r h}^{\prime}=m_{h r} \frac{N_{h}}{n^{2}} .
$$

Similarly as for matrix $\mathbf{G}$, the direct and indirect influence is calculated as in the following expression:

$$
\mathbf{M}^{*}=\mathbf{M}^{\prime}+\mathbf{M}^{\prime 2}+\mathbf{M}^{\prime 3}+\ldots
$$

The index of the centrality for the land use $h\left(I_{h}^{c}\right)$ is given by the sum by row minus the sum by columns, in other words the capacity to influence minus the degree of being influenced:

$$
I_{h}^{c}=\sum_{r} m_{h r}^{\star}-\sum_{h} m_{h r}^{\star}
$$

After having established a method for calculating an index of centrality we can forecast the relation between the optimal configuration and the self-organized dynamics. According to the results shown in table 5 (last column) all the efficient solutions generated with a big weight assigned to the retail land use should coincide with the result of the self-organized dynamics. In fact, 
$\boxplus$ Housing $\$$ Retail 贯 Industry $\square$ Open land $\square$ Equipment
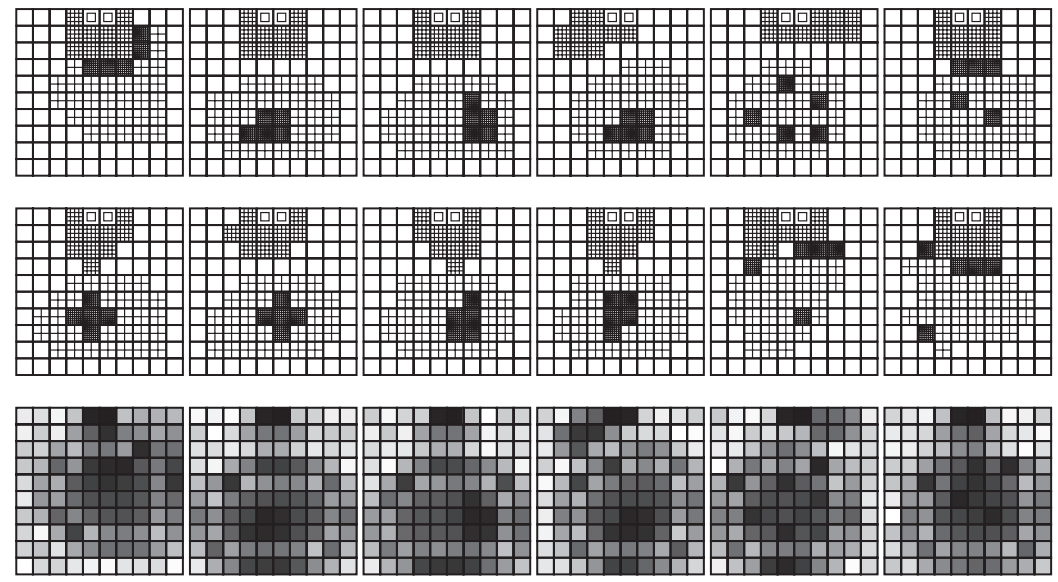

$\begin{array}{ll}W_{h}: & 0.05 \\ W_{r}: & 0.05 \\ W_{i}: & 0.90\end{array}$

\begin{abstract}
0.05
0.50

0.45
\end{abstract}

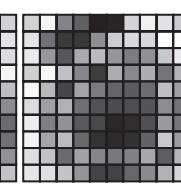

0.50
0.45
0.05

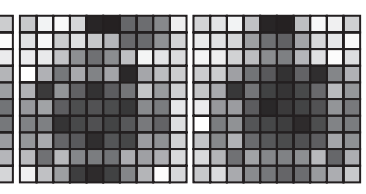

0.90

0.05

0.05
0.50

0.05

0.45

Fig. 12. The more realistic experiment. Upper row shows the patterns obtained through optimization. Middle row shows the patterns obtained by applying the rules of the self-organized dynamics to a random pattern. Bottom row shows the rank of pinning cells in relation to the influence index $\left(I^{i}\right)$. The gray scale represents the influence of the cells: the most influential cells are black, the least influential ones are white. At the bottom of each column the set of weights utilized for housing $\left(W_{h}\right)$, retail $\left(W_{r}\right)$ and industry $\left(W_{i}\right)$ is shown.

Table 5. Elements of the matrix $M^{*}$

\begin{tabular}{llllllr}
\hline Land use & Housing & Retail & Industry & Open land & $\begin{array}{c}\text { Equipment } \\
\begin{array}{c}\text { Centrality } \\
\text { index }\left(I^{c}\right)\end{array}\end{array}$ \\
\hline Housing & 0.09899 & 0.01983 & 0.00020 & 0 & 0 & -0.22033 \\
Retail & 0.13632 & 0.02316 & 0.01053 & 0 & 0 & 0.11671 \\
Industry & 0.03497 & 0.00851 & 0.01861 & 0 & 0 & -0.03929 \\
Open land & 0.06661 & 0.00120 & 0.00001 & 0 & 0 & 0.06782 \\
Equipment & 0.00247 & 0.00060 & 0.07202 & 0 & 0 & 0.07510 \\
\hline
\end{tabular}
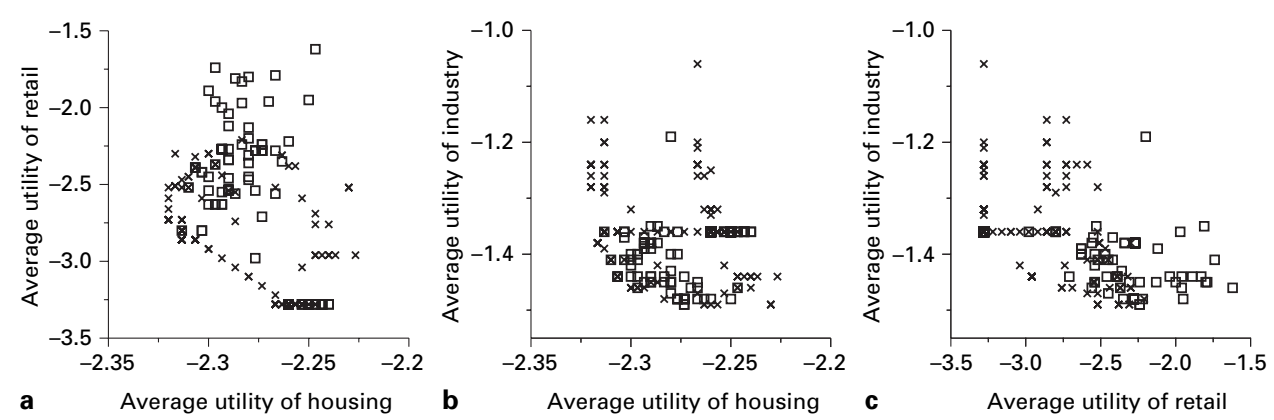

Fig. 13. The more realistic experiment. The Pareto front. $\times=$ Configurations obtained through optimization; $\square=$ configurations obtained by applying the rules of the self-organized dynamics to a random pattern.

\section{A $30 \times 30$ Grid Experiment}

Finally and in order to discuss the scalability of the proposed method the spatial grid has been enlarged to $30 \times 30$ squared cells. The rules of interactions included in the matrix $\mathbf{M}$ are the same unless the neighbourhood $\Phi_{i}$ is utilized to calculate the spatial relations, which has been enlarged to the 48 cells included in the square of $7 \times 7$ cells around the central cell in question. This enlargement is necessary in order to simulate the long-range spatial relations, and it is the only change which assures the scalability of the proposed method.

as figure 12 shows, the optimal configuration matches the self-organized dynamics when a big weight is assigned to the retail land use. In addition, the consequences of the establishment of pinning cells are shown in figure 14. The distance decreases quickly with the increase of the number of the pinning cells when the weight of the land uses and the centrality index does match (see fig. 14b, c). In case they do not, utilized to control the other land uses (fig. $14 \mathrm{a}, \mathrm{f}$, in which the first chosen pinning in this case the control of an only limited part of the pinning cells produces a convergence, with a limited amount of energy spent in the control. 

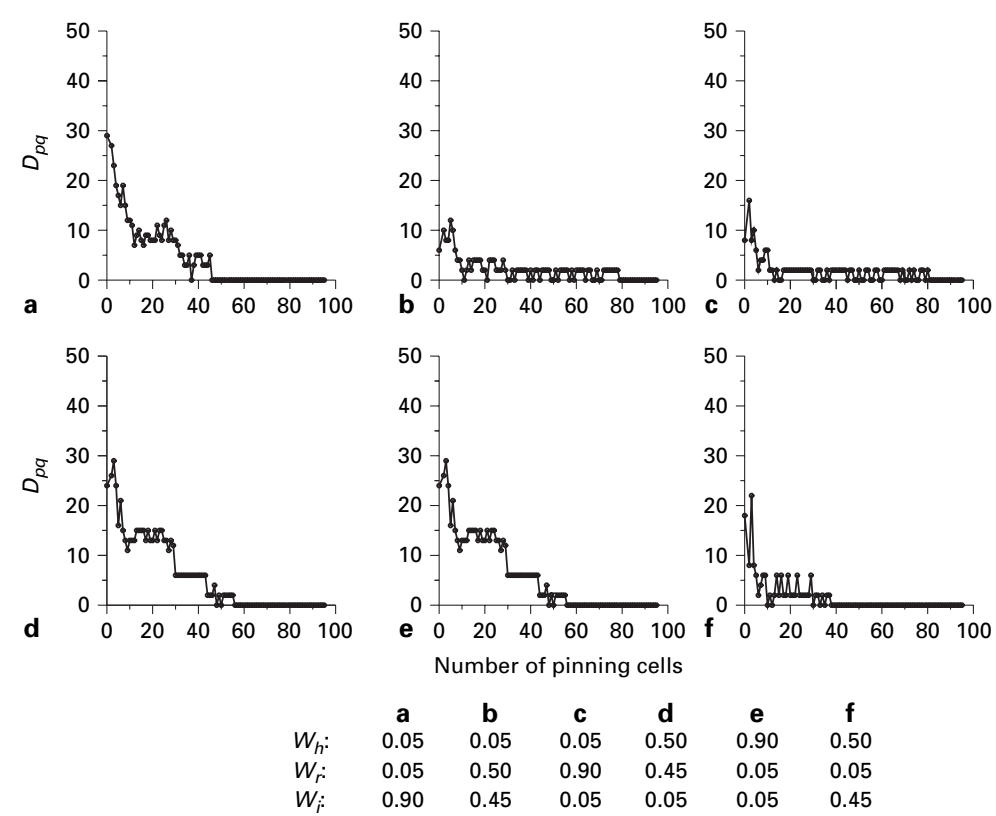

$\begin{array}{cccc}\mathbf{a} & \mathbf{b} & \mathbf{c} & \mathbf{d} \\ 0.05 & 0.05 & 0.05 & 0.5 \\ 0.05 & 0.50 & 0.90 & 0.45 \\ 0.90 & 0.45 & 0.05 & 0.05\end{array}$

Fig. 14. The more realistic experiment. The varying of distance $D_{p q}$ with the increase of the number of the pinning cells in which the land use has been fixed.

The number of land uses has been proportionally increased: housing: 270 cells, retail: 50 cells, industry: 90 cells, open land: 470 cells and equipment: 20 cells. Only two cases are shown (fig. 15) which utilize the same set of weight as the first and the sixth case shown in figure 12. The analysis of the convergence in relation to the number of the pinning cells is shown in figure 16. The results are similar to the $10 \times 10$ experiments, even if a finer tuning of the interaction matrix is requested in order to obtain a pattern less schematic and more similar to that observed in the reality.

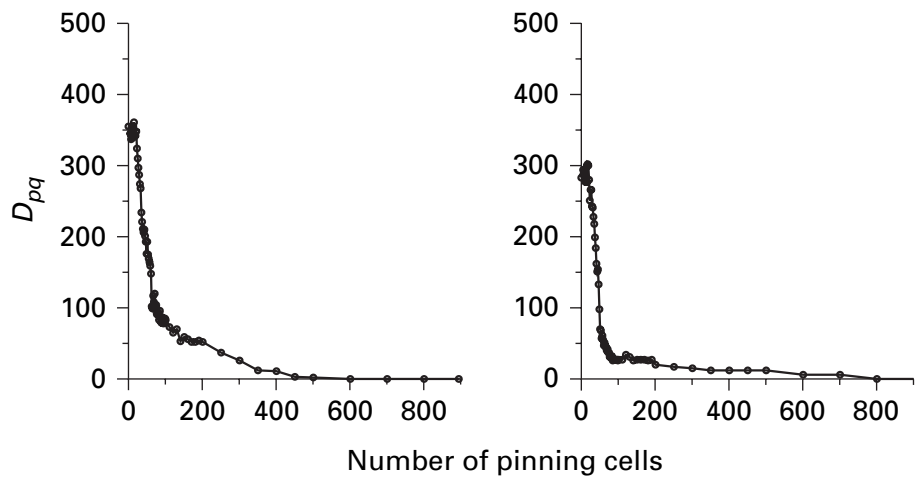

Fig. 16. The $30 \times 30$ grid experiment. The varying of distance $D_{p q}$ with the increase of the number of the pinning cells in which the land use has been fixed.
Relation with the Urban Planning and Control

In this experimental section we found that the optimization process and the selforganized dynamics produce similar results when the weight assigned to the land uses coincides with the index of centrality of land use. However, when this coincidence does not occur, the land use with a big index of centrality can be easily utilized to control the self-organized dynamics. These conclusions have to be related to some experience of urban planning especially in the fields of the regeneration of existing areas [17].In this case what planners are looking for is the catalyst effects of the urban project [21]. This is very similar to obtaining the desired plan with the control of a limited number of cells. The catalyst is in fact like a pinning cell in which planning effort is concentrated in order to stimulate the development in the desired direction. However, the identification of the critical point [22] where to concentrate investments is a further possible utilization of the proposed method. In this way the strict zoning control could be relaxed in order to allow the network of local actions to operate more freely.

\section{Conclusion}

We have shown that optimization and self-organized dynamics can be conceived as a similar process. On one hand, optimization is a special kind of dynamics, and on the other hand the self-organized dynamics is a special kind of optimization. In other words the urban systems are also problem solving. In turn the solving of the problem can be conceived as a dynamic process in which at each step the utility arising from each couple of bordering cells is considered instead of focusing on the utility of only one land use, and with the utilization of an exogenous factor, i.e. the cooling, which allows the system to reach and maintain the optimal configuration. Sometimes the attractors of the self-organized dynamics and of the optimization 
$\boxplus$ Housing
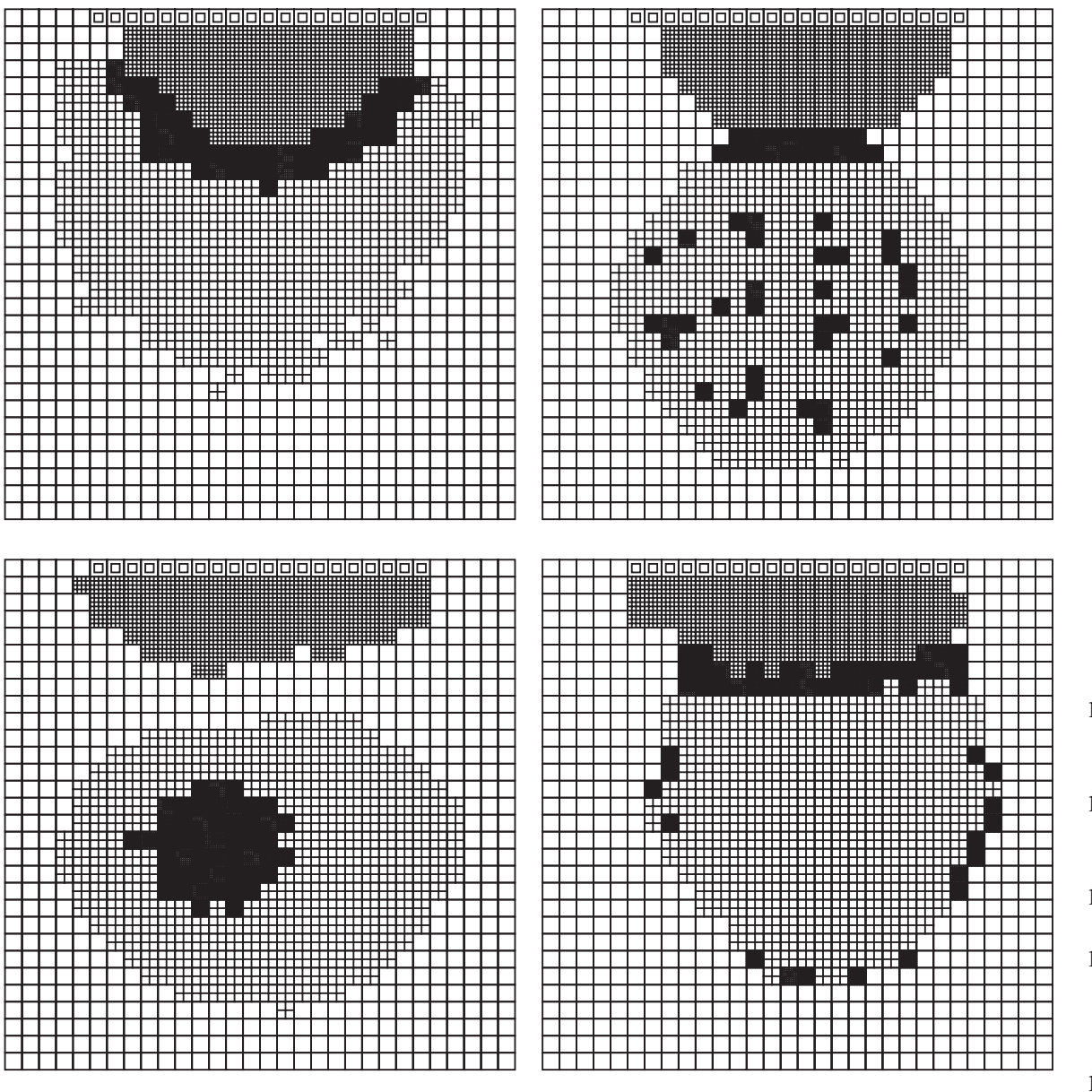

Fig. 15. The $30 \times 30$ grid experiment. Upper row shows the patterns obtained through optimization. Lower row shows the patterns obtained by applying the rules of the self-organized dynamics to a random pattern. The two columns of graphs refer to the following set of weights: first column: $W_{h}=0.05, W_{r}=0.05$, and $W_{i}=0.9$; second column: $W_{h}=0.5, W_{r}=0.05$, and $W_{i}=0.45$. They have to be compared with first and sixth cases in figure 12 .

process are similar. This is the Utopia in which individual and total utility do coincide. In case this does not happen, as usually, instead of using the control extended to each point of the surface that totally constraints the self-organized dynamics with the plan, we proposed a method which takes advantage of the self-organizing character of the urban dynamics and minimizes the controlling effort. Finally, this method can be applied to the urban planning practice based on the research of the

\section{References}

1 Portugali J: Self-Organization and the City. Berlin, Springer, 1999.

2 Cartwright TJ: Planning and chaos theory. J Am Plann Assoc 1991; 57: 44-56.

3 Alexander C: The Timeless Way of Building. New York, Oxford University Press, 1979.

4 Batty M: Cities, planning, design, computation and evolution. Environ Plann B 1995; 22: 379-382.

5 Portugali J, Alfasi N: Planning just-in-time versus planning just-in-case. Cities 2004; 21: 29-39.

6 Koopmans TC, Beckman MJ: Assignment problems in the location of economic activities. Econometrica 1957; 25: 53-76.

7 Schlager KJ: A land use plan design model. J Am Inst Plann 1965; 31: 103-111.

8 Bammi D, Bammi D: Development of comprehensive land use plan by means of a multiple objective mathematical programming model. Interfaces 1979; 9: 50-63.

-9 Michalek JJ, Choudhary R, Papalambros PY: Architectural layout design optimization. Eng Optimiz 2002; 34: 461-484.

10 Balling RJ, Taber JT, Brown MR, Day K: Multiobjective urban planning using genetic algorithm. J Urban Plan Dev Div 1999; 125: 86-99.

11 Cheng-Min F, Jen-Jia L: Using a genetic algorithm to generate alternative sketch maps for urban planning. Comput Environ Urban 1999; 23: 91-108.

12 Harris B: Optimization and design. Environ Plann B 1998(anniversary issue):23-28.

13 Fonseca CM, Fleming PJ: Multiobjective optimization; in Baeck T, Fogel D, Michalewicz Z (eds): Handbook of Evolutionary Computation. Oxford, IOP Publishing, 1998-2000.

14 Kirkpatrick S, Gelatt C, Vecchi M: Optimization by simulated annealing. Science 1983;220: 671-680.

15 Helbing D, Vicsek T: Optimal self-organization. New J Phys 1999; 1: 13.1-13.17.

16 Torrens PM, O'Sullivan D: Cellular automata and urban simulation: where do we go from here? Environ Plann B 2001; 28: 163-168.

17 Attoe W, Logan D: American Urban Architecture: Catalysts in the Design of Cities. Berkeley, University of California Press, 1991.

18 Grigoriev RO, Cross MC, Schuster HG: Pinning control of spatiotemporal chaos. Phys Rev Lett 1997; 79: 2795-2798.

element able to produce a positive catalytic effect on the whole urban structure.

\section{Acknowledgements}

I would like to thank Stefano Ruffo for stimulating discussions while this study was performed and Fabio Schoen for reviewing a previous version of the paper. The author is solely responsible for the opinions expressed.

19 Junge L, Parlitz U, Tasev Z, Kocarev L: Synchronization and control of spatially extended systems using sensor coupling. Int J Bifurcat Chaos 1999; 9: 2265-2270.

20 Schelling TC: Models of segregation. Am Econ Rev 1969; 59: 488-493.

21 Lowe M: The regional shopping centre in the inner city: a study of retail-led urban regeneration. Urban Stud 2005; 42: 449-470.

22 Chalkley B, Essex S: Urban development through hosting international events: a history of the Olympic games. Planning Perspect 1999; 14: 369-394. 